\title{
Identification of a prognostic three-long noncoding RNA signature in lung squamous cell carcinoma via bioinformatic analysis
}

\author{
Rongjiong Zheng ( $\nabla$ 842429171@qq.com ) \\ Ningbo Yinzhou No 2 Hospital \\ Mengdi Zheng \\ Ningbo Yinzhou No 2 Hospital \\ Mingming Wang \\ Ningbo Yinzhou No 2 Hospital https://orcid.org/0000-0003-2808-5889 \\ Meiling Hu \\ Cixi People's Hospital
}

\section{Research}

Keywords: lung squamous cell carcinoma, LncRNAs, Survival

Posted Date: August 20th, 2020

DOI: https://doi.org/10.21203/rs.3.rs-60427/v1

License: (a) (i) This work is licensed under a Creative Commons Attribution 4.0 International License. Read Full License 


\section{Abstract \\ Background}

Lung squamous cell carcinoma (LSCC) is a form of cancer that is associated with high rates of relapse, poor responsiveness to therapy, and a relatively poor prognosis. The relationship between long noncoding RNA (IncRNA) expression and LSCC patient prognosis remains to be established.

\section{Methods}

In the present study, we discovered that IncRNAs were differentially expressed in LSCC tumor tissues relative to normal control tissues, and we explored the prognostic relevance of these IncRNA expression patterns using data from the Cancer Genome Atlas (TCGA).

\section{Results}

These multidimensional data were analyzed in order to identify IncRNA signatures that were associated with LSCC patient survival outcomes. Kaplan-Meier survival curves revealed prognostic capabilities for three of these IncRNAs (LINC02555, APCDD1L-DT and OTX2-AS1). A Cox regression analysis revealed this three-IncRNA signature to be significantly associated with patient survival. Further GO and KEGG analyses revealed that the predicted target genes of these three IncRNAs were also potentially involved in cancer-associated pathways.

\section{Conclusions}

Together these results thus indicate that this novel three-IncRNA signature can be used to predict LSCC patient prognosis.

\section{Background}

Lung cancer is a highly heterogeneous disease, with genetic, epigenetic, and environmental factors all acting to shape its development and progression. Lung cancer mortality rates are the highest of all forms of cancer, accounting for $25 \%$ and $30 \%$ of all cancer-associated deaths in the USA and China, respectively [1-2]. In 2015 alone, 733,000 new cases of lung cancer were diagnosed in China (69\% in males and 31\% in females), while 218,527 new cases were diagnosed in the USA during this same period ( $52 \%$ in males and $48 \%$ in females). SEER data indicate that lung cancer patients exhibit a 5 -year survival rate of just $18.1 \%$ [3]. Lung squamous cell carcinoma (LSCC) cases account for a significant fraction of overall lung cancer cases [4]. LSCC more often occurs in men, is related to the smoking of tobacco, and is often associated with high rates of relapse, poor responsiveness to therapeutic intervention, and a generally poor patient prognosis [5-6]. While there have been many advances in the field of clinical oncology as a 
whole in recent years, rates of 5-year overall survival (OS) for LSCC patients still remain low. As such, it is vital that novel approaches be identified that can be used to predict the prognosis of LSCC patients so as to guide clinical decision making and treatment efforts in these individuals.

Long non-coding RNAs (IncRNAs) are RNA molecules > 200 nucleotides in length that lack coding potential [7] Emerging evidence indicates that some IncRNAs do encode proteins and play roles in transcriptional, and epigenetic gene regulation, and cancer [8-9]. These IncRNAs have been shown to frequently be dysregulated in cancer, with their altered expression patterns having a direct impact on tumor cell gene expression at the post-transcriptional and epigenetic levels, as well as on the proliferation, survival, invasion, and metastasis of these cells [10-12]. However, relatively few studies to date have specifically examined the relationship between IncRNA expression and LSCC patient prognosis. In the present study, we therefore explored patterns of differential IncRNA expression in LSCC tumor tissues and normal control tissue samples in an effort to assess the prognostic relevance of such IncRNA expression patterns. Through this approach we were able to develop a three IncRNA signature which was found to be significantly associated with the survival of LSCC patients.

\section{Materials And Methods}

\section{LSCC patient datasets}

Level 3 expression and clinical data pertaining to 432 LSCC patients and 49 control samples were downloaded from The Cancer Genome Atlas (TCGA, https://tvga-data.nci.nih.gov/tcga/). Datasets and patient records were used in order to assess both patterns of IncRNA expression as well as clinocpathological and demographic variables including gender, age at time of diagnosis, and TNM staging (Table 1). The Ethics Committee of the Institutional Review Board of Ningbo Yinzhou Second Hospital and Cixi People's Hospital in Zhejiang Province approved this study. Samples were included in the present analysis if they were from patients with an OS > 1 month for whom IncRNA differential expression data and information pertaining to clinical details and prognosis were available. The language package in $\mathrm{R}$ was used in order to interpret the IncRNA sequencing data, while the limma package was used when assessing differential IncRNA expression between LSCC and control samples, with differential expression being expressed based upon fold change (FC) values. Those IncRNAs with a $\log _{2}|\mathrm{FC}|>1.0$ and $p<0.05$ were considered to be significantly differentially expressed.

\section{Statistical analysis}

The prognostic relevance of differentially expressed IncRNAs in LSCC was assessed using Kaplan-Meier curves and log-rank tests. We ultimately constructed a signature using a linear combination of the expression levels of these three IncRNAs and the estimated regression coefficients in the multivariate Cox regression analysis. This three IncRNA signature-derived risk score was then used to stratify patients into high- and low-risk groups, using the median risk score in this cohort as a cutoff point for stratification purposes. Kaplan-Meier curves and log-rank tests were then used to compare survival outcomes between 
these high- and low-risk patients. In addition, receiver operating characteristic (ROC) analyses were used in order to compare the sensitivity and specificity of this three IncRNA risk score as a means of predicting patient survival outcomes. $\mathrm{P}<0.05$ was the significance threshold. R version 3.5.1 (http:// www.rproject.org/) was used for all statistical testing [13].

\section{Functional analysis}

Correlating genes to the differentially expressed IncRNAs were obtained using the co-expression method. Pearson correlation coefficients between the expression profiles of the three prognostic IncRNAs and their protein-coding genes (PCGs) were calculated to determine their relationships. Those PCGs with a Pearson's $\mathrm{R}>0.40$ and $p<0.05$ were considered to be IncRNA-related. These putative IncRNA targets were then subjected to Gene Ontology (GO) and Kyoto Encyclopedia of Genes and Genomes (KEGG) functional enrichment analyses. Furthermore, these target genes were incorporated into a protein-protein interaction (PPI) network using the STRING database [14], with Cytoscape being used for network visualization [15]. Select protein pairs from this network with $>10$ nodes were the outputs of this analysis.

\section{Results}

\section{Patient characteristics}

The study investigated 481 patient samples, including 432 LSCC and 49 normal tissues. Table 1 lists detailed clinical characteristics, including gender, race, age at diagnosis, and disease stage. Of the enrolled patients, $26.2 \%$ were female, and $78.4 \%$ were order than 60 years. The most common tumor grades were I (49.3\%) and II (32.4\%). A total of 936 differentially expressed IncRNAs, including 687 upregulated and 249 downregulated IncRNAs, were identified between LSCC and normal tissues in Figure 1.

\section{The relationship between IncRNA expression and LSCC patient OS}

We began by using univariate and multivariate Cox proportional hazard regression models in order to identify those IncRNAs which were associated with LSCC patient prognosis. In total, we identified three candidate IncRNAs in these LSCC patients ( $p<0.01$; Fig 2). A multivariate model confirmed that the expression of the IncRNAs LINC02555 (HR = 1.08, $p=0.025)$, APCDD1L-DT (HR = 1.10, $p=0.004)$, and OTX2-AS1 (HR $=0.89, p=0.006)$ were all independently associated with LSCC patient OS. Kaplan-Meier survival curves and log-rank tests were further used to examine the relationship between these IncRNAs and patient survival. We found that two of the tested IncRNAs (LINC02555 and APCDD1L-DT) were negatively associated with LSCC patient OS, whereas the IncRNA OTX2-AS1 was positively correlated with OS (Fig. 3).

\section{The prognostic utility of the three IncRNA signature}

Using this three IncRNA signature, we were able to assign risk scores to patient samples, after which these samples were separated into high- and low-risk groups based upon the median risk score value $(n=$ 
216 samples/group). We then found that patients in the high-risk group had a significantly shorter OS than did patients in the low-risk group $(p<0.001)$ (Fig 4a). We then used an ROC analysis in order to assess the prognostic utility of this three IncRNA signature. The AUC values for these curves as predictors of LSCC patient 3- and 5-year survival were 0.67 and 0.62 , respectively, corresponding to an effective survival prediction (Fig 4b). Patients in the high-risk group expressed higher levels of the IncRNAs LINC02555 and APCDD1L-DT on average relative to low risk patients, whereas low-risk patients expressed higher levels of OTX2-AS1 IncRNA.

\section{Functional enrichment analysis and PPI networks}

In order to identify potential targets for these three IncRNAs which were associated with LSCC patient prognosis, we conducted a co-expression analysis as detailed in the Materials and Methods section. We then performed GO and KEGG pathway analyses on these co-expressed genes in order to unravel their potential physiological roles (Fig 5). These co-expressed genes were primarily enriched in genes associated with biological processes such cell adhesion molecule binding, ubiquitin-like protein ligase binding, protein serine/threonine kinase activity, ubiquitin protein ligase binding, actin binding, ATPase activity, phospholipid binding, and phosphoric ester hydrolase activity. These genes were additionally significantly enriched in KEGG pathways including endocytosis, focal adhesion, MAPK signaling, lysosomes, neurotrophin signaling, ubiquitin mediated proteolysis, axon guidance, herpes simplex virus 1 infection, and the cell cycle. Furthermore, PPI networks were also obtained using the STRING tool. As mentioned in Fig 5c, INS, GCG, GCGR, GLP1R, IAPP, P2RY1, CRH and FFAR1 had the most connections with other members of the module and were thus the most noteworthy nodes in this network.

\section{Discussions}

Lung cancer currently ranks as the deadliest form of cancer, and as such it is a primary focus for many cancer research efforts [16]. While LSCC patient prognosis has improved significantly in recent years owing to improvements in multidisciplinary treatment strategies, and chemotherapeutic/radiotherapeutic treatment regimens, LSCC recurrence rates remain high and as such this disease can impose a heavy burden upon patients, their families, and on medical institutions [17-18]. Difficulties in accurately diagnosing LSCC and in predicting patient outcomes have led to low 5-year survival rates in affected patients [2]. Recent work suggests that the ability to more reliably predict LSCC patient prognosis at time of initial diagnosis is associated with a significant improvement in patient outcomes. As such, it is vital that novel biomarkers that can reliably predict LSCC patient outcomes be identified. It is similarly important that the molecular mechanisms governing the development and progression of LSCC be fully elucidated.

Many studies have clearly shown that the development of LSCC can be driven by interactions between genetic, transcriptomic, and proteomic factors [19-20]. Changes in IncRNA expression patterns can also influence all stages of the oncogenic process, yet the prognostic relevance of these IncRNAs has not been sufficiently studied to date. As such, in the present study we examined IncRNA expression patterns in 
LSCC and were thus able to identify three IncRNAs that were significantly linked with LSCC patient OS. These three IncRNAs were then subjected to additional analyses aimed at identifying their putative target genes and potential biological roles through the use of pathway enrichment analyses. These results indicated that these three IncRNAs may play roles in regulating LSCC molecular pathogenesis, clinical progression, and patient prognosis, thus clearly demonstrating the prognostic relevance of IncRNA expression patterns in LSCC patients in a clinical setting.

Multiple studies [21-22] have demonstrated that functional IncRNA expression can modulate oncognesis via altered regulation of gene expression and signaling within tumor cells. Indeed, certain IncRNAs are able to promote the development, progression, and metastasis of tumors through their ability to regulate the proliferation, differentiation, migration, and survival of these cancerous cells [23]. Huang et al. [24] found that increasing the expression of the downregulated IncRNA LINC00961 resulted in increased Bax expression and the corresponding apoptotic death of NSCLC cells. Xu et al. further provided evidence suggesting that the IncRNA HULC is able to promote LSCC cell proliferation owing to its ability to PTPROdependent phosphorylation and activation of NF-KB [25]. Similarly, Wang et al. found that increased expression of the IncRNA MIR31HG in NSCLC led to enhanced tumor cell gefitinib resistance owing to associated activation of the EGFR/PI3K/AKT signaling pathway. In this report, we analyzed highthroughput data and were thereby able to identify two upregulated IncRNAs (LINC02555 and APCDD1LDT) and one downregulated IncRNA (OTX2-AS1) in LSCC patients, all three of which were significantly associated with patient clinical outcomes.

We further sought to gain insight into the functional importance of the three IncRNAs identified in this study via using a co-expression analysis-based approach to identify putative IncRNA target genes that were then subjected to GO and KEGG enrichment analyses. This approach revealed the IncRNAassociated target genes to be enriched for functionality in the context of endocytosis, focal adhesion, MAPK signaling, and lysosomal activity, all of which are closely linked with oncogenesis and tumor progression [27-28]. To date no studies have specifically studied LINC02555, APCDD1L-DT, or OTX2-AS1 in the context of LSCC. As such, future in-depth molecular analyses will be needed to confirm the findings of our predictive co-expression analysis.

There are multiple limitations to the present study. For one, these results are derived solely from bioinformatics analyses and as such necessitate additional functional validation. Furthermore, we did not explore the molecular mechanisms linking the expression of these three IncRNAs to LSCC patient prognosis, and as such future experimental studies will be required in order to elucidate these mechanisms. As such, large-scale multi-center trials will be essential in order to validate and expand upon our findings.

\section{Conclusions:}

In summary, in the present article we were able to identify three different IncRNAs that could be used to predict survival outcomes in patients with LSCC. Further large-scale multi-center trials will be needed to 
confirm our findings, and to explore the molecular mechanisms linking these IncRNAs to clinical outcomes in LSCC patients. While much work is still required before this IncRNA signature can be implemented in a clinical setting, we nonetheless feel that our findings may have significant value as a future diagnostic or prognostic tool in the context of LSCC patient identification and care.

\section{Abbreviations}

LSCC: lung squamous cell carcinoma; LncRNAs:Long non-coding RNAs; TCGA:The Cancer Genome Atlas; PCGs:Protein-coding genes; ROC:Receiver operating characteristic; OS:Overall survival; FCs:Fold changes; KEGG:Kyoto Encyclopedia of Genes and Genomes; GO:Gene Ontology; BP:Biological process; NFKB:nuclear factor-кB

\section{Declarations}

\section{Funding}

None

\section{Ethics approval and consent to participant}

The study was performed in accordance with the guidelines of the Declaration of Helsinki. The study protocol was approved by the Ethics Committee of the Institutional Review Board of the Cixi People's Hospital and Ningbo Yinzhou Second Hospital.

\section{Consent for publication}

Not applicable

\section{Competing Interests}

No competing interests exist.

\section{Data availability statement}

All data are fully available without restriction.

\section{Authors' contributions}

RJZ and MLH carried out the study design, analysis and interpretation of data. RJZ, MMW and MDZ drafted the manuscript. All read and approved the final manuscript.

\section{Acknowledgements}

Special thanks to all participants who took part in this study. 


\section{References}

1. American Thoracic Society/European Respiratory Society International Multidisciplinary Consensus Classification of the Idiopathic Interstitial Pneumonias. This joint statement of the American Thoracic Society (ATS), and the European Respiratory Society (ERS) was adopted by the ATS board of directors, June 2001 and by the ERS Executive Committee, June 2001. Am J Respir Crit Care Med. 2002;165:277-304.

2. Chen W, Zheng R, Baade PD, Zhang S, Zeng H, Bray F, Jemal A, Yu XQ, He J. Cancer statistics in China, 2015, CA: a cancer journal for clinicians, 66 (2016) 115-132.

3. Mayer JE, Swetter SM, Fu T, et al. Screening, early detection, education, and trends for melanoma: current status (2007-2013) and future directions: Part II. Screening, education, and future directions. J Am Acad Dermatol. 2014 Oct;71(4):611. ..e1-611.e10; quiz 621-2.

4. Travis WD, Brambilla E, Noguchi M, et al. International association for the study of lung cancer/american thoracic society/european respiratory society international multidisciplinary classification of lung adenocarcinoma. J Thorac Oncol. 2011 Feb;6(2):244-85.

5. Kenfield SA, Wei EK, Stampfer MJ, et al. Comparison of aspects of smoking among the four histological types of lung cancer. Tob Control. 2008 Jun;17(3):198-204.

6. Antal CE, Hudson AM, Kang E, et al. Cancer-associated protein kinase $C$ mutations reveal kinase's role as tumor suppressor. Cell. 2015 Jan 29;160(3):489-502.

7. Carpenter S. Long noncoding RNA: Novel links between gene expression and innate immunity. Virus Res 137-145, 2016.

8. $\mathrm{Li} \mathrm{CH}$, Chen Y. Insight Into the Role of long noncoding RNA in cancer development and progression. Int Rev Cell Mol Biol 33-65, 2016.

9. Morris KV, Mattick JS. The rise of regulatory RNA. Nat Rev Genet. 2014 Jun;15(6):423-37.

10. Zhang B, Dong Y, Zhao Z. LncRNA MEG8 regulates vascular smooth muscle cell proliferation, migration and apoptosis by targeting PPARa. Biochem Biophys Res Commun. 2019 Feb 26;510(1):171-176.

11. Huang Y, Ni R, Wang J, et al. Knockdown of IncRNA DLX6-AS1 inhibits cell proliferation, migration and invasion while promotes apoptosis by downregulating PRR11 expression and upregulating miR144 in non-small cell lung cancer. Biomed Pharmacother. 2019 Jan;109:1851-9.

12. Dong $X$, Chen R, Lin H, et al. IncRNA BG981369 Inhibits Cell Proliferation, Migration, and Invasion, and Promotes Cell Apoptosis by SRY-Related High-Mobility Group Box 4 (SOX4) Signaling Pathway in Human Gastric Cancer. Med Sci Monit. 2018 Feb;5:24:718-26.

13. Team RC. A language and environment for statistical computing. Vienna: R Foundation for Statistical Computing; 2014.

14. Szklarczyk D, Franceschini A, Wyder S, Forslund K, Heller D, Huerta-Cepas J, et al. STRING v10: protein-protein interaction networks, integrated over the tree of life. Nucleic Acids Res. 2015;43:44752. 
15. Shannon P, Markiel A, Ozier O, Baliga NS, Wang JT, Ramage D, et al. Cytoscape: a software environment for integratedmodels of biomolecular interaction networks. Genome Res. 2003;13:2498-504.

16. Torre LA, Siegel RL, Jemal A. Lung Cancer Statistics. Adv Exp Med Biol. 2016;893:1-19.

17. Hirsch FR, Scagliotti GV, Mulshine JL, et al. Lung cancer: current therapies and new targeted treatments. Lancet. 2017 Jan 21;389(10066):299-311.

18. Lemjabbar-Alaoui $\mathrm{H}$, Hassan OU, Yang YW, et al. Lung cancer: Biology and treatment options. Biochim Biophys Acta. 2015 Dec;1856(2):189-210.

19. Denisov EV, Schegoleva AA, Gervas PA, et al. Premalignant lesions of squamous cell carcinoma of the lung: The molecular make-up and factors affecting their progression. Lung Cancer. 2019 Sep;135:21-8.

20. Prelaj A, Tay R, Ferrara R, et al. Predictive biomarkers of response for immune checkpoint inhibitors in non-small-cell lung cancer. Eur J Cancer. 2019 Jan;106:144-59.

21. Li Y, Han X, Feng H, et al. Long noncoding RNA OIP5-AS1 in cancer. Clin Chim Acta. 2019 Aug 30;499:75-80.

22. Wang M, Zhou L, Yu F, et al. The functional roles of exosomal long non-coding RNAs in cancer. Cell Mol Life Sci. 2019 Jun;76(11):2059-76.

23. Wang M, Zhou L, Yu F, Zhang Y, Li P, Wang K. The functional roles of exosomal long non-coding RNAs in cancer. Cell Mol Life Sci. 2019;11:2059-76.

24. Huang Z, Lei W, Tan J, et al. Long noncoding RNA LINC00961 inhibits cell proliferation and induces cell apoptosis in human non-small cell lung cancer. J Cell Biochem. 2018 Nov;119(11):9072-80.

25. Xu Y, Li J, Wang P, et al. LncRNA HULC promotes lung squamous cell carcinoma by regulating PTPRO via NF-KB. J Cell Biochem. 2019 Aug 25.

26. Wang B, Jiang H, Wang L, Chen X, Wu K, Zhang S, Ma S, Xia B. 2017. Increased MIR31HG IncRNA expression increases gefitinib resistance in non-small cell lung cancer cell lines through the EGFR/PI3K/AKT signaling pathway. Oncol Lett. 133494 - 500.

27. Wu DM, Wang YJ, Han XR, et al. LncRNA LINC00880 promotes cell proliferation, migration, and invasion while inhibiting apoptosis by targeting CACNG5 through the MAPK signaling pathway in spinal cord ependymoma. J Cell Physiol. 2018 Sep;233(9):6689-704.

28. Schlienger S, Ramirez RA, Claing A. ARF1 regulates adhesion of MDA-MB-231 invasive breast cancer cells through formation of focal adhesions. Cell Signal. 2015 Mar;27(3):403-15.

\section{Tables}

Table1. Baseline characteristics of all patient samples 


\begin{tabular}{|c|c|c|}
\hline \multirow[t]{2}{*}{ Variables } & \multicolumn{2}{|c|}{ All samples $(n=481)$} \\
\hline & No. & $\%$ \\
\hline \multicolumn{3}{|l|}{ Gender } \\
\hline Female & 126 & 26.2 \\
\hline Male & 355 & 73.8 \\
\hline \multicolumn{3}{|c|}{ Age at diagnosis } \\
\hline$>60$ & 377 & 78.4 \\
\hline$\leq 60$ & 104 & 21.6 \\
\hline \multicolumn{3}{|l|}{ Stage } \\
\hline I & 237 & 49.3 \\
\hline II & 156 & 32.4 \\
\hline III & 81 & 16.8 \\
\hline IV & 7 & 1.5 \\
\hline \multicolumn{3}{|l|}{ T stage } \\
\hline $\mathrm{T} 1$ & 110 & 22.9 \\
\hline T2 & 282 & 58.6 \\
\hline T3 & 68 & 14.1 \\
\hline $\mathrm{T} 4$ & 21 & 4.4 \\
\hline \multicolumn{3}{|c|}{ Metastasis } \\
\hline MO & 398 & 82.7 \\
\hline M1 & 7 & 1.5 \\
\hline$M X$ & 76 & 15.8 \\
\hline \multicolumn{3}{|c|}{ Lymph node status } \\
\hline NO & 307 & 63.8 \\
\hline $\mathrm{N} 1$ & 125 & 26.0 \\
\hline N2 & 38 & 7.9 \\
\hline N3 & 5 & 1.1 \\
\hline NX & 6 & 1.2 \\
\hline
\end{tabular}


Figures

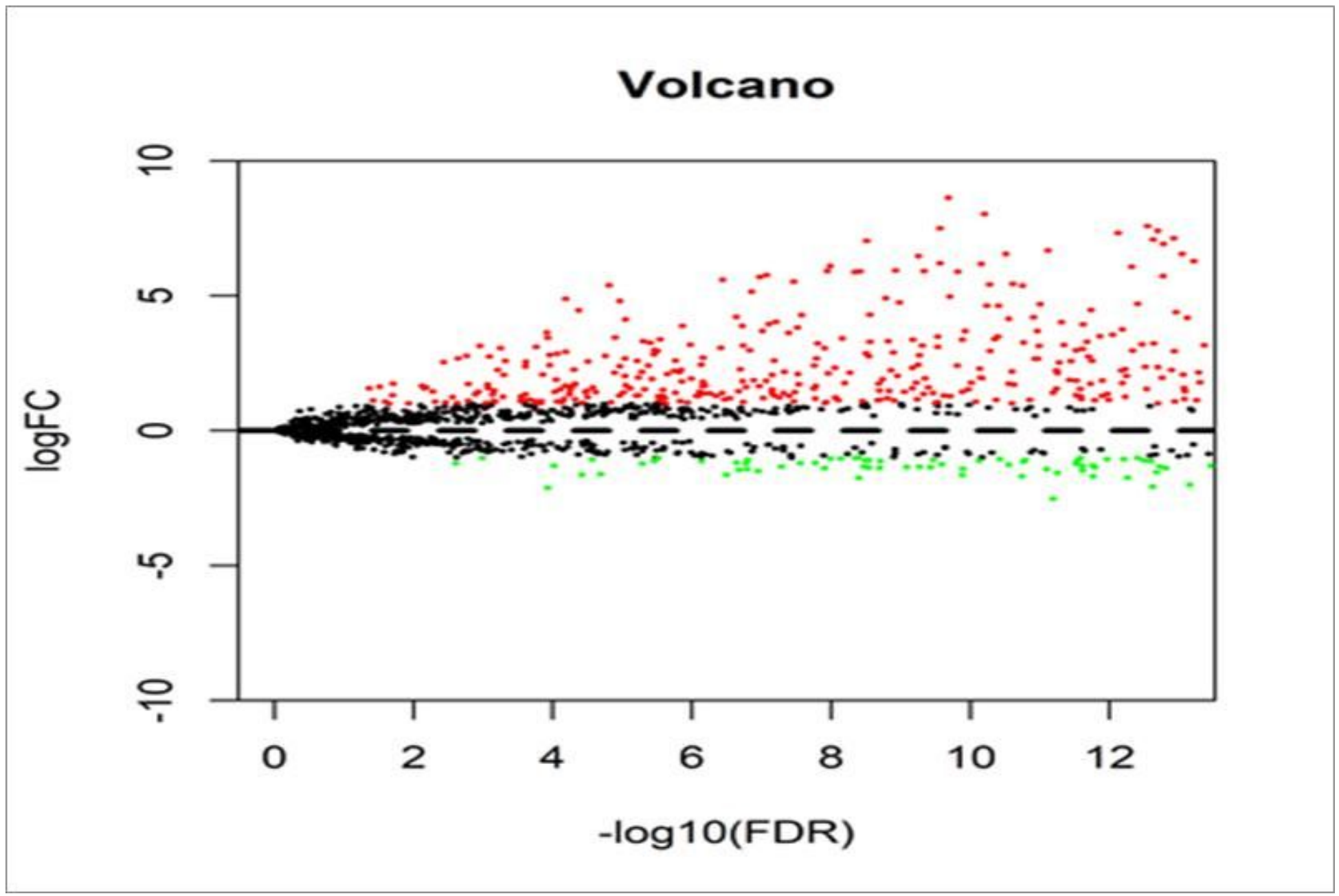

Figure 1

Volcano plot of differentially expressed IncRNAs. Red and green dots represent upregulated and downregulated IncRNAs, respectively. 


\section{Hazard ratio}

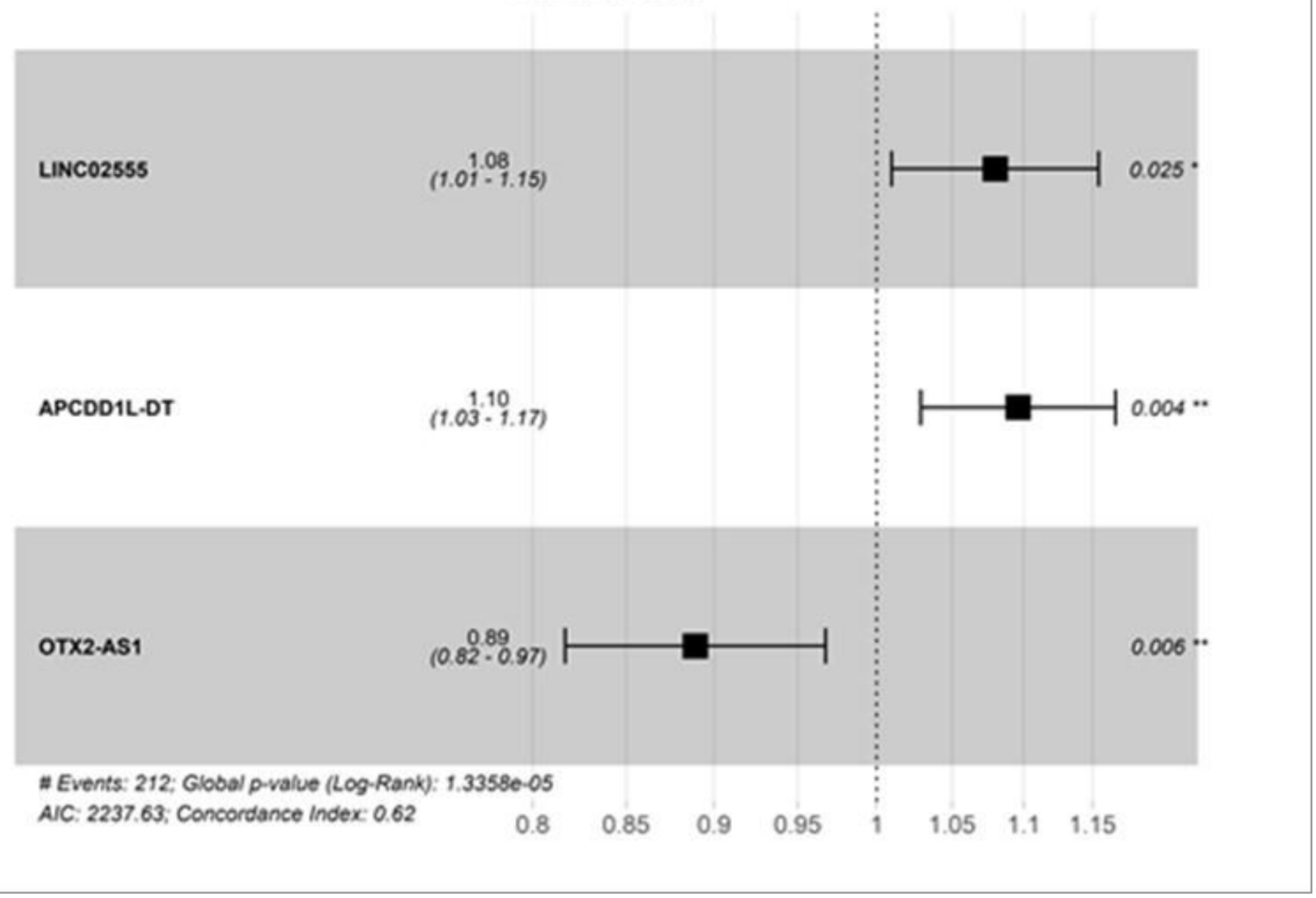

\section{Figure 2}

Forest plot for the association between three IncRNAs and risk value. ${ }^{\star} p<0.05,{ }^{\star \star} p<0.01$ 
a

Survival curve (p=3.036e-03)

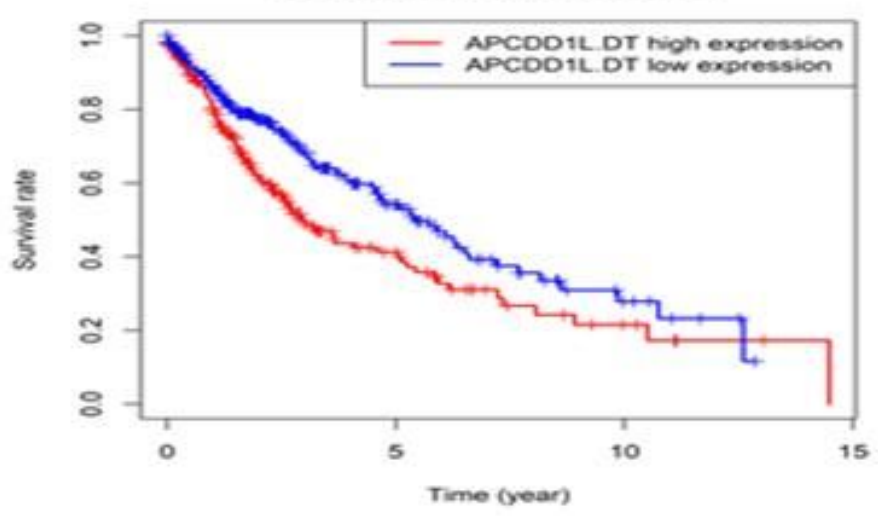

C

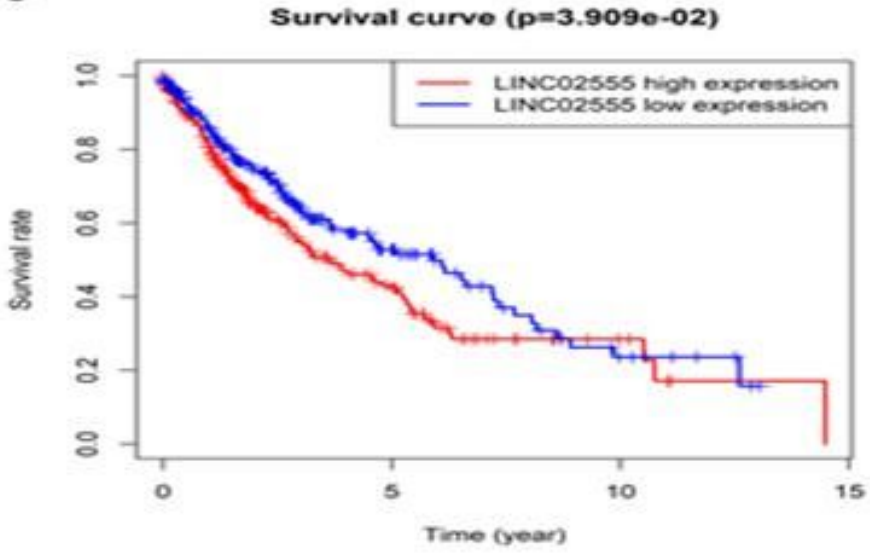

b

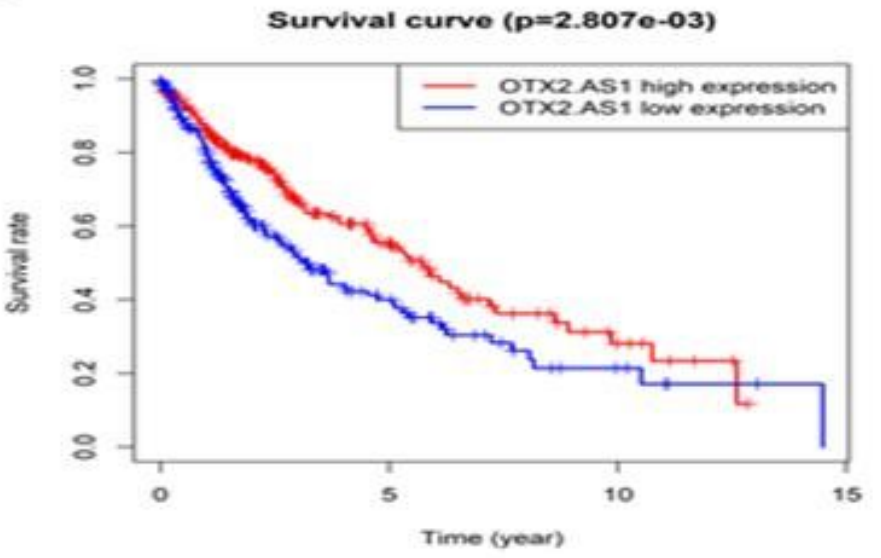

\section{Figure 3}

Kaplan-Meier method and log-rank test revealed that three IncRNAs were associated with OS in patients with LSCC. The patients were divided into low and high expression levels group according to the median value. (A) APCDD1L-DT (B) OTX2-AS1 and (C) LINC02555 
a

Survival curve $(p=2.6530-03)$

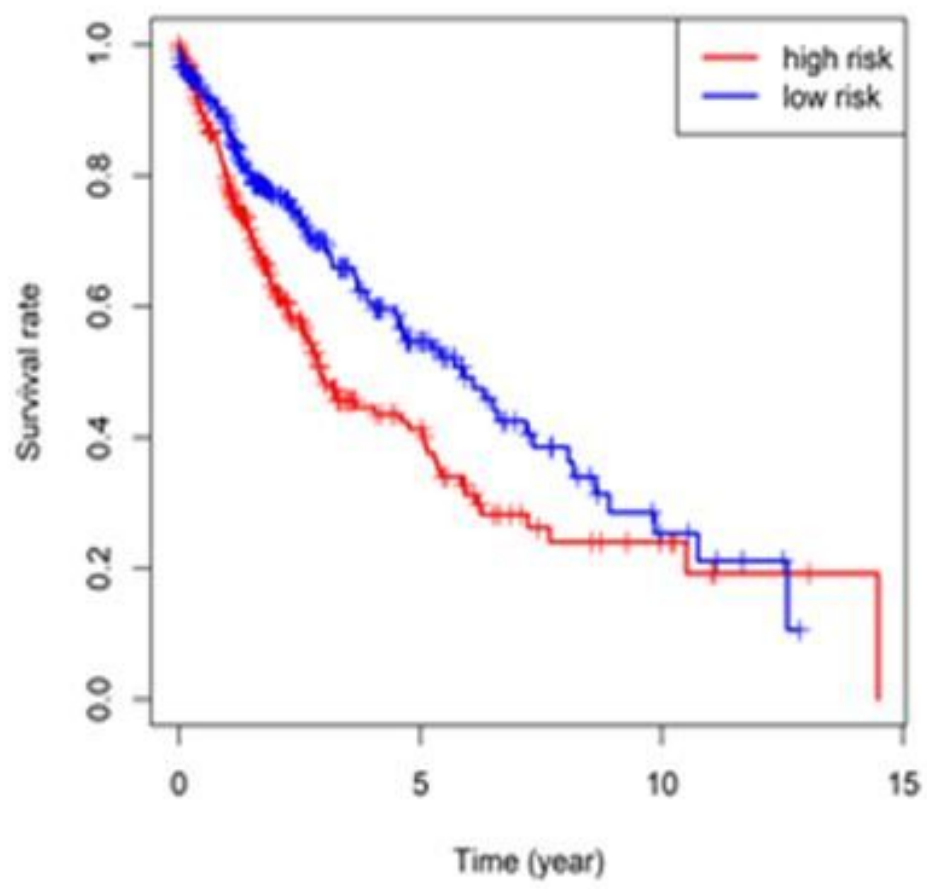

b

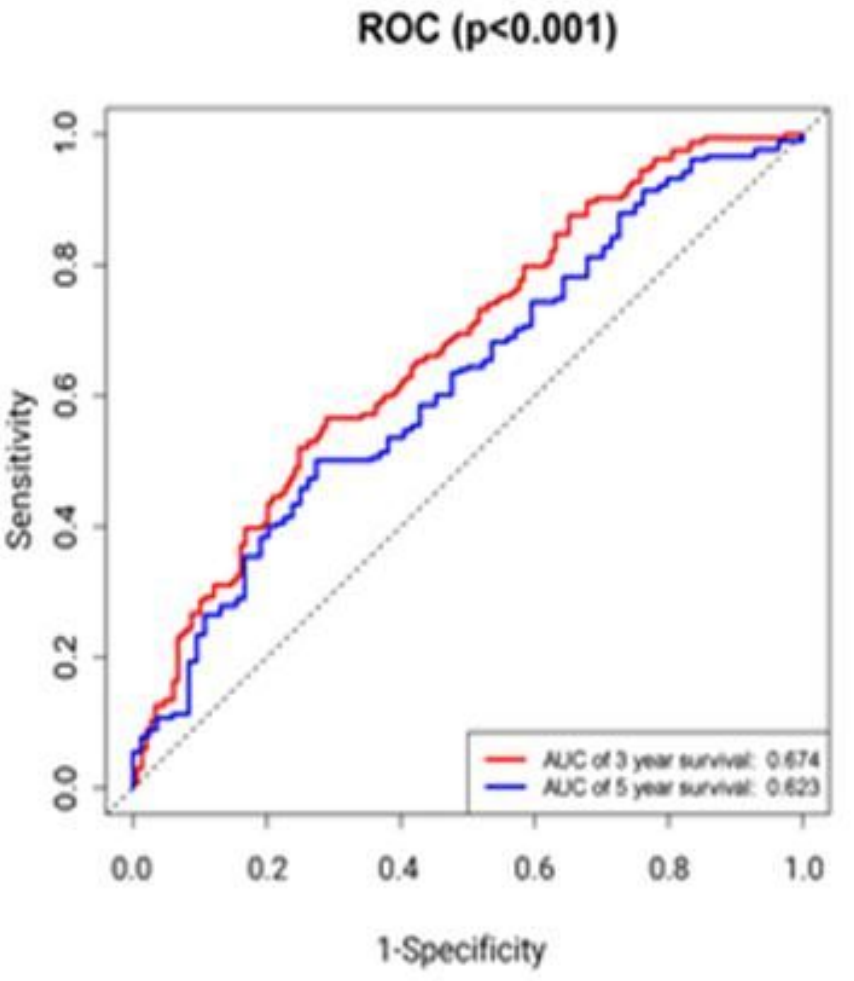

\section{Figure 4}

(a) Kaplan-Meier estimates of the survival outcomes for patients using the three-IncRNA signature, and

(b) receiver operating characteristic analysis of risk factors for survival prediction 
a

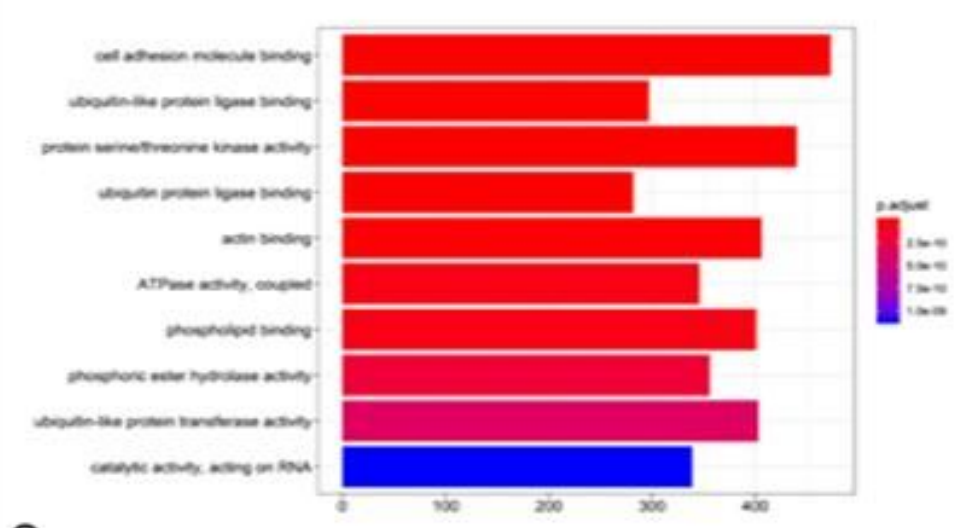

C

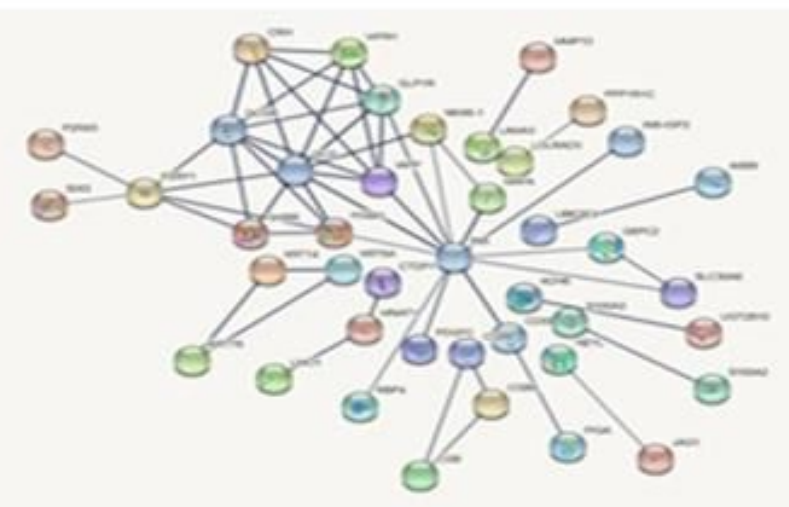

b

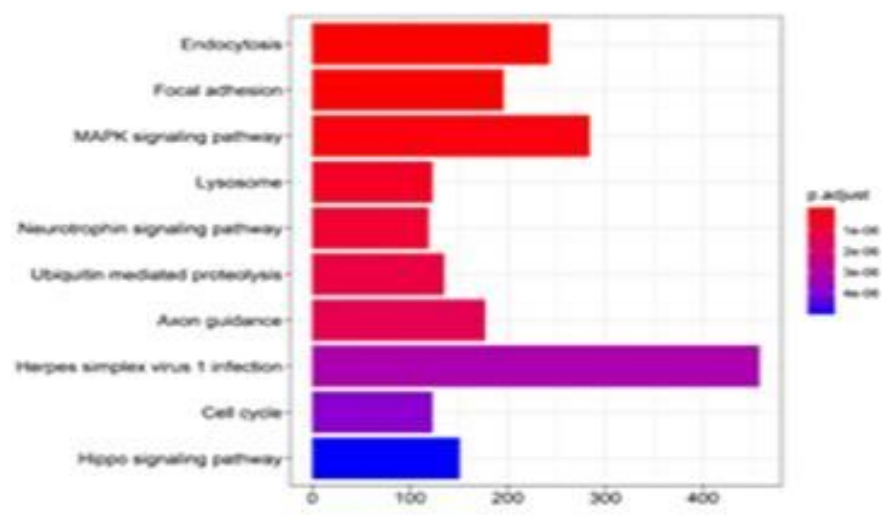

Figure 5

(a) The Gene Ontology and (b) Kyoto Encyclopedia of Genes and Genomes pathway enrichment analyses, and (c) PPI networks 